\title{
Globale Gesundheitssteuerung und globale Steuerung für mehr Gesundheit
}

\section{RÜDIGER KRECH}

Dr. Rüdiger Krech ist Direktor im Büro der Stellvertretenden Generaldirektorin für Gesundheitssysteme und Innovation der Weltgesundheitsorganisation (WHO) in Genf

\author{
Die Rolle der Internationalen Organisationen und die \\ globale Steuerung von Prozessen und Entscheidungen, die \\ Auswirkungen auf die globale Gesundheit haben, sollten \\ neu beleuchtet werden. Der vorliegende Beitrag skizziert \\ die Gründe und untersucht die Rolle und Aktivitäten \\ der wichtigsten "globalen Spieler". Eine wesentliche \\ Schlussfolgerung ist, dass dringend ein klares Regelwerk \\ sowohl für die globale Gesundheitssteuerung als auch für \\ die globale Steuerung für mehr Gesundheit benötigt wird.
}

Es gibt mindestens fünf gewichte Gründe, warum die Rolle der Internationalen Organisationen und die globale Steuerung von Prozessen und Entscheidungen, die Auswirkungen auf die globale Gesundheit haben, neu beleuchtet werden müssen:

1. Der Gesundheitssektor hat längst nicht mehr die alleinige Kontrolle über Gesundheit: Mulitlaterale Verhandlungen zu Themen der Öffentlichen Gesundheit werden aus mehreren Gründen immer komplexer: Zum einen liegt dies daran, dass Gesundheit von multiplen Faktoren beeinflusst wird. So hängt die Zunahme von nicht übertragbaren, chronischen Erkrankungen nicht nur von demografischen Einflüssen ab, sondern auch davon, wie viel oder wie wenig Menschen sich bewegen, was sie essen, wie gesund sie leben, wie sich ihre Lebensumstände gestalten und unter welchen sozialen, wirtschaftlichen und kulturellen Bedingungen sie leben ${ }^{\mathrm{I}}$. Diese gesellschaftlichen Bedingungen sind weltweit aber auch innerhalb von Staaten sehr ungleich und führen dazu, dass viele Menschen sehr viel höhere Krankheits- und Sterberisiken haben als andere. Lebensweisen und gesellschaftliche Bedingungen werden wiederum von vielen Politikbereichen sowie beispielsweise durch grosse Wirtschaftskonzerne beeinflusst und hängen somit zum Grossteil von Entscheidungen ab, die ausserhalb des Gesundheitssektors gemacht werden. Zum anderen sind Politikentscheidungen, die die Lebensbedingungen der Menschen beeinflussen, immer enger miteinander verzahnt. So hat der globale Umgang mit Antibiotika in der Tiermassenhaltung als Fleischproduktionsbooster Einfluss auf die Antibiotikaresistenz beim Menschen ${ }^{\text {II }}$ oder aber der globale Temperaturanstieg durch CO2 Emissionen Auswirkungen auf die Ausbreitung von Malariagebie-

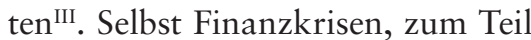
durch die derzeitigen Arbeitsweisen im Investmentbanking und im Hochfrequenzhandel ausgelöst, können mit spezifischen Krankheitsindikatoren und Sterberaten assoziiert werden und fordern heute schätzungsweise weltweit etwa 280.000 Menschenleben im $\mathrm{Jahr}^{\mathrm{IV}}$. Deshalb nimmt die Bedeutung der Arbeit an den sozialen Determinanten - also den Bedingungen unter denen Menschen geboren werden, in denen sie aufwachsen, leben, arbeiten und altern - in der globalisierten Welt 
von heute zu. Zu den Aktionsebenen gehören neben einer besser abgestimmten und ressortübergreifenden Regierungsführung, einer besser umgesetzten Bürgerbeteiligung und einer Reform des Gesundheitssystems (die bspw. die neue Rolle der Interessensvermittlung zwischen unterschiedlichen Sektoren und Akteuren besser versteht) auch eine wesentlich engere internationale Zusammenarbeit zwischen denjenigen Organisationen, die Entscheidungen mit Gesundheitsrelevanz treffen ${ }^{\mathrm{V}}$.

2. Der Privatsektor spielt eine immer grössere Rolle: Die Intensivierung der Interaktionen, Verbindungen und gegenseitigen Abhängigkeiten zwischen Staaten, Gesellschaften und Unternehmen und die Auswirkungen der sich daraus ergebenen kommerziellen Interessen auf die Öffentliche Gesundheit, die ich als "kommerzielle Determinanten von Gesundheit" bezeichnen möchte, müssen in den $\mathrm{Ge}$ sundheitswissenschaften noch viel besser analysiert und verstanden werden. Die global agierenden Unternehmen im Nahrungsmittelbereich oder auch die Softdrink-, Alkohol- und Tabakproduzierenden Industriebetriebe und ihre Interaktionen untereinander, aber auch mit nationalen, regionalen und internationalen Organisationen wären sicher ein lohnendes Forschungsfeld für die Gesundheitswissenschaften.

3. Keine Gesellschaft der Welt wird die Kosten bestreiten können, die in den nächsten zwanzig Jahren entstehen, um allein die medizinische Versorgung von Patienten mit nicht übertragbaren, chronischen Erkrankungen zu gewährleisten $^{V I}$. So kommt eine Studie der Weltbank und des Weltwirtschaftsforums aus dem Jahr $2011 \mathrm{zu}$ dem Schluss, dass diese Kosten dann $48 \%$ des Weltbruttoinlandsproduktes von 2010 entsprechen würden. Das wiederum hat die Politik alarmiert: Gesundheit hat auf der politischen Agenda in den letzten Jahren erheblich an Wichtigkeit gewonnen. Viele Regierungschefs haben die Rolle von Gesundheit im Hinblick auf Gesamtentwicklung, gesellschaftliche Stabilität und Frieden erkannt. Nicht nur in den Vereinigten Staaten von Amerika, sondern auch in Brasilien, Kenia oder Chile werden Wahlen mit Gesundheitsthemen gewonnen oder verloren.
4. Gesundheit ist zu einer Industrie geworden: Gesundheit ist heute einer der grössten Wirtschaftszweige der Welt und ebenfalls einer ihrer grössten Arbeitgeber. Insoweit hat der Gesundheitssektor global erheblich zu einer Stabilisierung der Wirtschaften in der globalen Finanzkrise 2008 beigetragen. In den letzten zehn Jahren haben sich die globalen Gesundheitsausgaben von etwa 3 of 6.5 Billionen US Dollar (eine 6 mit 12 Nullen; in der

\section{Globale Gesundheitssteuerung (health governance)}

Alle der oben genannten Organisationen haben sich grösseren Reformprozessen unterzogen oder sind gerade dabei, dies zu tun. Sie haben ihren Fokus geschärft, haben zum Teil, wie die WHO, ihren Personalkörper verkleinert und begonnen, wirkungsorientierter zu arbeiten. Die Aufsichtsgremien der Organisationen, in denen in allen Fällen Vetreter der selben Mitgliedsländer und Partner sitzen, haben diese Prozesse eng begleitet. Interessanter Weise hat die Frage einer Koordination der alten und neu entstandenen Organisationen untereinander in diesen Diskussionen bislang eine sehr geringe Rolle gespielt. So gibt es kein Gremium, das sich übergeordnet mit der (Neu-) Verteilung der Mandate der amerikanischen Zählweise 6.5 Trillion USD) mehr als verdoppelt. ${ }^{1}$ VII

5. Auf der globalen Ebene gibt es immer mehr Gesundheitsorganisationen: Die führenden Internationalen Organisationen im Bereich der Öffentlichen Gesundheit sind neben der Weltgesundheitsorganisation (WHO), Unicef, UNFPA und UNAIDS. Neben diesen "H4" gibt es eine erweitere Gruppe "H8” zu denen zusätzlich der Global Fund to Fight Aids, Tuberculosis and Malaria (GFATM), the Global Alliance for Vaccines and Immunization (GAVI), die Weltbank (WB) und die Bill\&Melinda Gates Foundation gehören.

Es gibt also zwei Problembereiche, die einer genaueren Betrachtung bedürfen: Auf der einen Seite geht es um die verbesserte Koordination der alten und neu entstandenen Organisationen, die sich auf der globalen Ebene mit Gesundheit beschäftigen. Es geht zum anderen darum, die Einflüsse von Entscheidungen auf der globalen Ebene, die Auswirkungen auf die globale Gesundheit haben, im Vorfeld besser zu verstehen, den Entscheidungsträgern zu verdeutlichen und "das Thema Gesundheit" in solche Entscheidungsprozesse mit einzubeziehen. Der erste Problembereich lässt sich im Englischen gut mit "health governance" beschreiben, der zweite mit dem Begriff "governance for health". einzelnen Organisationen und globalen Partnerschaften beschäftigt.

An dieser Stelle sei ein kurzer Exkurs zu der Finanzierung dieser Gesundheitsorganisationen gestattet, denn diese Organisationen verfügen mitnichten über die gleichen Finanzmittel: So entspricht das Budget der WHO mit ihren sechs Regionalbüros und ihren etwa 120 Länderbüros zusammen etwa dem Budget von zwei grösseren Universitätskrankenhäusern in Deutschland ${ }^{2}$. Das verausgabte Gesamtbudget des Globalen Fonds zur Bekämpfung von Aids, Tuberkulose und Malaria lag 2012 bei etwa 23.8 Milliarden USD. Deutschland hat im November 2013 auf der 4. Auffüllungskonferenz, die ein Gesamtvolumen von 12 Milliarden USD (und damit etwas über ein Dreifaches des Zweijahresbudgets der WHO) erbrachte, eine Zahlungszusage von 800 Millionen USD für einen Zeitraum von 2014-16 zugesagt ${ }^{\mathrm{VIII}}$. "The Global Alliance for Vaccines and Immunization (GAVI)" hat in den 12 Jahren seit der Gründung etwas mehr als 7.6 Milliarden USD als Spenden

1 Bei dieser Entwicklung muss man allerdings den schwächeren US Dollarkurs mit einrechnen.

2 Das Jahresbudget des UKE beträgt laut Geschäftsbericht 2011 http:// www.uke.de/medien/downloads/ gb-unternehmenskommunikation/ Geschaeftsbericht 2011.pdf, S. 55, 763 Mio Euro (etwa 991 Mio USD). Das zweijahresbudget der WHO liegt bei 3.9 Mrd. USD. http://www.who.int/about/ resources_planning/A66_R2_en.pdf 
erhalten ${ }^{\mathrm{IX}}$ und davon die Impfung von 370 Millionen Kindern in 72 Ländern finanziert. Das Jahresbudget wird 2014 bei etwa 1.6 Milliarden USD liegen. Ende 2013 lagen weitere Zahlungszusagen von den Partnern der Impfallianz bei etwa 5 Milliarden USD. Für UNAIDS wird das Budget für den Zeitraum 2014-15 bei 485 Millionen USD liegen, was etwa $50 \%$ der reinen Mitgliedsbeiträge ${ }^{3}$ der WHO entspricht. Im Zweijahreszeitraum 2011-2012 vergab die die Weltbankgruppe Kredite im Gesundheitssektor von ca. 10.6 Milliarden USD ${ }^{\mathrm{x}}$.

Eins der Ziele der WHO Reform ist eine grössere Kohärenz in der globalen Gesundheitssteuerung. Dabei kommt der WHO eine Führungsrolle zu „um die unterschiedlichen Spieler und Partner zu befähigen einen effektiven Beitrag zur Weltgesundheit zu leisten“. Aber welche Mechanismen hat das Sekretariat der WHO, um dieses Ziel umzusetzen? Im Bericht der WHO Generaldirektorin an den Aufsichtsrat zur Rolle der WHO in der globalen Gesundheitssteuerung XI geht sie in Paragraph 23 genauer darauf ein, beschränkt sich aber darauf, dass neue Partnerschaften und Initiativen einen klaren Mehrwert zeigen müssten. Wie eine übergeordnete Koordinationsstruktur, die bspw. Redundanzen in der fachlichen Arbeit aufdeckt und möglichst schon im Vorfeld verhindert, eine Arbeitsteilung zwischen den Organisationen und Partnerschaften verbindlich festlegt und überwacht, ist bislang nicht Teil der offiziellen Diskussion, um bestehende

\section{Ohne eine verbesserte Koordina- tion der Gesundheitsarbeit auf der globalen Ebene wird es in Zukunft mehr Krankheiten geben - nicht nur in Entwick- lungsländern, sondern weltweit.}

\section{Globale Steuerung für mehr Gesundheit (governance for health)}

Dies gilt noch mehr für den Bereich, der sich, wie oben beschrieben, mit den politischen und kommerziellen Determinanten von Gesundheit beschäftigen muss. Es gibt derzeit keine Funktion in der WHO, die die Diskussionen innerhalb der unterschiedlichen internationalen Organisationen, die Einfluss auf die globale Gesundheit haben werden, mitschneidet. Wenn in der FAO Entscheidungen zu $\mathrm{X}$, oder in der Welthandelsorganisation Entscheidungen zu Y getroffen werden, werden die Konsequenzen, die diese Entscheidungen auf Gesundglobale Dysfunktionen in der weltweiten Gesundheitssteuerung anzugehen.

Ilona Kickbusch stellte ihrem Gutachten zur Positionierung Deutschlands in der globalen Gesundheitspolitik ${ }^{\mathrm{XII}}$ ein Zitat von James Rosenau voran: „We are living in a messy world“. Das stimmt zweifelsohne für die globale Gesundheit, wie auch der Versuch einer Kartierung der Auswirkungen der Arbeit dieser Organisationen berücksichtigt.

Kohärenzbemühungen sind nicht nur im Bereich „Gesundheit“ vonnöten. „Ernährung“ kann zum Nachdenken anregen: Die Nahrungsmittelkrise, die vor allem arme Menschen in Entwicklungsländern 2008 in grosse Not gestürzt hat, führte beispielsweise auf Weisung des Aufsichtsrats der UN (Chief heit haben werden, derzeit nicht wirklich
Executive Board) zu einer hochrangigen Arbeitsgruppe zwischen Weltbank, Internationalem Währungsfonds, der OECD und der Welthandelsorganisation, um zukünftigen Nahrungsmittelkrisen vorzubeugen. Die „UN High Level Task Force on the Food Security Crisis" ist allerdings eine fachliche Arbeitsgruppe mit keinerlei politischer Aufsichtsfunktion. Die Ergebnisse der Arbeitsgruppe beschränken sich denn auch auf „Empfehlungen“ ohne bindende Wirkung. Sie entwickelte 2008 einen umfassenden Aktionsrahmen für Ernährungssicherheit (Comprehensive Framework of Action on Food Security) ${ }^{\mathrm{XIV}}$. Parallel wurde vom Vereinigten Königreich und Frankreich während des G8 Gipfels 2008 eine Partnerschaft ins Leben gerufen, die sich „Global Partnership on Agriculture, Food Security and Nutrition" nennt. Nachdem diese dann zunächst nicht arbeitsfähig war, wurde der Vorschlag 2009 erneut auf die Tagesordnung der G8 gesetzt. Diesmal gab es Mittelzusagen von 20 Milliarden USD, wovon allerdings lediglich 6 Mrd. neues Geld waren. 2012 wurde diese Partnerschaft „erneuert“, um die Privatwirtschaft an dieser Partnerschaft zu beteiligen. Eine Studie von Oxfam im September 2013 bescheinigt dieser Partnerschaft jedoch wenig Erfolg ${ }^{X V}$. Dieses Beispiel wird hier erwähnt, weil es ein bestimmtes Handlungsmuster internationaler Diplomatie widerspiegelt: Man

3 Also ohne freiwillige Beiträge 
reagiert politisch auf Krisen, indem man eine UN Facharbeitsgruppe etabliert, die allerdings keine Aufsichtsfunktion hat. Mit guten Intentionen will man dann schnell Ergebnisse sehen, die man eher von Partnerschaften als von bestehenden Organisationen erwartet. Mit wechselnden Krisen bleibt zwar das politische Engagement, aber es hapert, vor allem aufgrund systemischer Unzulänglichkeiten, an der Umsetzung vor Ort.

Es scheint, als würde man mit Instrumenten des 20. Jahrhunderts auf $\mathrm{He}-$ rausforderungen des 21. Jahrhunderts reagieren. Denn es ist längst deutlich, dass den negativen Auswirkungen, die Globalisierung neben ihren vielen positiven Seiten eben auch hat, nur mit effektiverer globaler Steuerung begegnet werden kann

Es braucht dringend ein klares Regelwerk sowohl für die globale „Gesundheitssteuerung “ als auch für die „globale Steuerung für mehr Gesundheit“. Passiert dies nicht, wird es mehr übergewichtige Menschen geben, die an chronischen Erkrankungen leiden. Es werden Infektionskrankheiten wieder vorkommen, die längst als ausgerottet galten. Letztendlich wird weiterhin der globale Gesundheitssektor die Kosten zu tragen haben, die durch Entscheidungen in anderen Politikbereichen produziert werden.

Und da dies von den Gesundheitssystemen in der derzeitigen Struktur wahrscheinlich nicht mehr vollständig finanziert werden kann, wird dies rückschliessend Auswirkungen auf die Qualität der Gesundheitsversorgung haben. Deshalb braucht es nach der Reform der internationalen Gesundheitsinstitutionen, die, wenn sie beendet ist, zu einer besseren globalen Gesundheitssteuerung beiträgt, eine weitere Reform. Der erste Schritt war und ist notwendig, um die globalen Institutionen für die Zukunft „fit zu machen“. Der zweite Schritt wird nötig sein, um gemeinsam letztendlich mehr Gesundheit zu produzieren. Diese weitere Reform sollte dann zu einer besseren „globalen Steuerung für mehr Gesundheit" führen. Davon ist man allerdings, wie oben geschildert, bislang noch ein gutes Stück entfernt. Die Rechenschaftspflichten der internationalen Akteure sind nicht geklärt. Noch gibt es keine Organisation auf globaler Ebene, die die Auswirkungen von im Entscheidungsprozess befindlichen Politiken auf Gesundheit analysiert, um die globale
Gesundheit zu schützen und zu fördern. Artikel 2 der Verfassung der WHO mag Ankerpunkte für ein solches Mandat liefern ${ }^{\mathrm{XVI}}$, aber es bedarf sicher einer wesentlich pointierter geführten Diskussion, um diesen zweiten wichtigen Reformschritt in den unterschiedlichen Organisationen, die gesundheitsrelevante Entscheidungen treffen, zu gehen.

\section{Literatur}

I Dahlgren G, Whitehead M. Policies and strategies to promote social equity in health. Stockholm: Institute for Future Studies; 1991.

II http://www.euro.who.int/_data/assets/ pdf_file/0005/136454/e94889.pdf

III http://www.who.int/globalchange/ climate/summary/en/index6.html

IV Williams, C; Zeltner, T; Maruthappu, M: "The Impact of economic recession on health outcomes: A Global Analysis, 1981-2011, unpublished paper

$\checkmark$ http://www.who.int/sdhconference/ declaration/Rio_political declaration.pdf

VI http://www3.weforum.org/docs/WEF Harvard HE GlobalEconomicBurden NonCommunicableDiseases 2011.pdf

VII http://apps.who.int/gb/ebwha/pdf files/ EB132/B132_26-en.pdf, page 7, para 17

VIII http://www.theglobalfund. org/en/mediacenter/ announcements/2013-12-04 Germany Voices_Consistent_Support_for_the Global Fund/

IX GAVI Alliance Progress Report 2012, S. 38 , accessed through: http://www.gavialliance.org/results/gavi-progress-reports/

X http://siteresources.worldbank.org/ EXTANNREP2013/Resources/93048871377201212378/9305896-1377544753431/ OpSumLendingTables_EN.pdf

XII http://apps.who.int/gb/ebwha/pdf files/ EB132/B132_5Add5-en.pdf

XII http://tablet. bundesgesundheitsministerium.de/ fileadmin/dateien/Downloads/I/ Internationale Gesundheitspolitik/ Kickbusch - Die Positionierung Deutschlands_in_der_globalen Gesundheitspolitik.pdf

XIII Privates Power Point slide von Ilona Kickbusch

XIV http://www.boell.de/sites/default/files/ Global-Governance-for-World-FoodSecurity.pdf, Seite 7

XV http://www.oxfam.org/sites/www. oxfam.org/files/bn-new-alliance-newdirection-agriculture-250913-en.pdf

XVI http://www.admin.ch/opc/de/classifiedcompilation/19460131/200906250000/ o.810.1.pdf

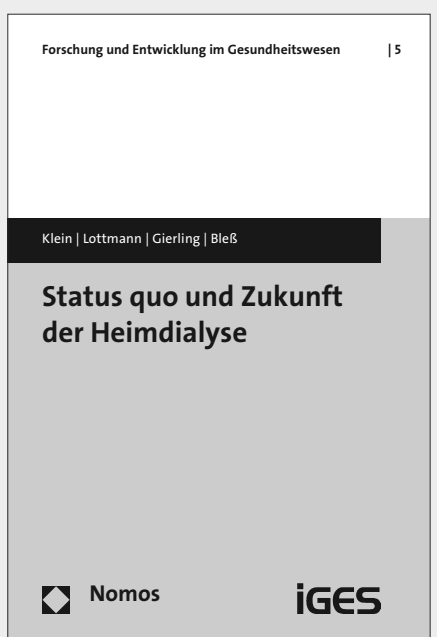

\section{Status quo und Zukunft der Heimdialyse}

Von Silvia Klein, Kathrin Lottmann, Patrick Gierling und Hans-Holger Bleß

2014, 200 S., brosch., 39,- $€$

ISBN 978-3-8487-1246-5

(Forschung und Entwicklung im Gesundheitswesen, $B d$. 5)

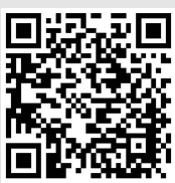

In dem Gutachten des IGES Instituts werden die derzeitige Dialyseversorgung in Deutschland analysiert und Handlungsoptionen zur Deckung des steigenden Versorgungsbedarfs diskutiert. Heimverfahren spielen dabei eine wichtige Rolle, da die Autoren eine Zunahme dialysepflichtiger Patienten bei gleichzeitigem Rückgang ambulant tätiger Nephrologen prognostizieren.

Portofreie Buch-Bestellungen unter www.nomos-shop.de/22507 Alle Preise inkl. Mehrwertsteuer Nomos 